\title{
Towards an Autonomous Evolution of Non-Biological Physical Organisms
}

\author{
Roderich Groß, Stéphane Magnenat, Lorenz Küchler, \\ Vasili Massaras, Michael Bonani, and Francesco Mondada \\ Ecole Polytechnique Fédérale de Lausanne, LSRO, Station 9, \\ CH-1015 Lausanne, Switzerland; roderich.gross@ieee.org
}

\begin{abstract}
We propose an experimental study where simplistic organisms rise from inanimate matter and evolve solely through physical interactions. These organisms are composed of three types of macroscopic building blocks floating in an agitated medium. The dynamism of the medium allows the blocks to physically bind with and disband from each other. This results in the emergence of organisms and their reproduction. The process is governed solely by the building blocks' local interactions in the absence of any blueprint or central command. We demonstrate the feasibility of our approach by realistic computer simulations and a hardware prototype. Our results suggest that an autonomous evolution of non-biological organisms can be realized in human-designed environments and, potentially, in natural environments as well.
\end{abstract}

Key words: Adaptation, artificial life, evolution, evolutionary robotics, morphology, origin of life, self-assembly, self-organization, self-replication

\section{Introduction}

In this paper, we argue that artificial evolution of living organisms could, or should, take place in worlds that obey the laws of physics, and where possible, in the natural world. If this were the case, the evolutionary processes would not only be validated but could explore deeply the world's own dynamics and its nonlinear nature [1]. A step towards this direction was made by Floreano and Mondada [2], who proposed an approach to evolve - without human intervention - the brain of a robot that interacts with its physical world. The brain, an artificial neural network, was modeled in software, but as Thompson [3] showed, it could be embedded into an electronic circuit as well. Sims [4,5] investigated computer simulations to evolve both body and brain of organisms (see also [6]). Funes and Pollack [7] investigated computer simulations to evolve static support structures made of realistic components (LEGO bricks), which allowed them to build and test the best solutions in reality. Lipson and Pollack [8] extended this approach by an automatic procedure to manufacture the solutions, in this case, robotic lifeforms.

Different from natural evolution, the aforementioned approaches to artificial evolution are not to the extent self-organized as we would like them to be. 
For example, they all share a central computer algorithm that decides whether organisms might reproduce or not. Moreover, they make use of dedicated computer algorithms that can produce new organisms on demand, for example, by recombining or varying existing solutions. By contrast, natural evolution is an autonomous, decentralized, and self-organized process that is fully embedded into the physical world. To the best of our knowledge, up to now the study of self-organized evolutionary processes has considered only fairly abstract models, where the genotypes (and genetic operators) are either software entities lacking embodiment [9-14] or entities having a rudimentary embodiment only [15].

Our work builds on recent advances in systems capable of macroscopic selfassembly [16,17]. In these systems, as the result of a self-organized process, a set of centimeter-sized building blocks can form composite entities. Several macroscopic self-assembly systems - ranging from purely mechanical parts to fully autonomous robots - proved capable of replicating connected composite entities [18-22]. However, these composite entities did not undergo change, and thus could not evolve (but see [23]). By contrast, we investigate a self-assembling system that is capable of producing a population of embodied organisms (i) which are subject to change through artificial evolution, and (ii) which respond to stimuli in their environment. The defining characteristics of our system are:

1. it is composed of pre-existing building blocks: energy modules, interaction modules, and boundary modules;

2. the modules (and composite entities) float passively in an agitated medium;

3 . the energy and interaction modules self-assemble into composite entities;

4. the energy modules transform and store energy provided by the environment;

5. the interaction modules respond to stimuli in their environment;

6 . the boundary modules attach to composite entities and thereby form protecting membranes, which inhibit further growth;

7. modules within a same composite entity share their energy;

8. modules without energy are not powered and can thus not actively bind with other modules (however, they can passively bind with active modules);

9. composite entities with an intact membrane replicate by self-assembly;

10. composite entities can break into multiple parts.

In the following we refer to composite entities with an intact membrane as organisms. Note that organisms (i) need energy, for example to maintain their connectivity (see item 8), and (ii) can replicate (see item 9). In this study, the organism (phenotype) is identical to the genotype.

The paper is organized as follows. Section 2 describes the simulator that we use to model the physical process. Section 3 explains the process itself. Sections 4 and 5 detail respectively the computational results and a hardware prototype. Section 6 discusses the findings and concludes the paper.

\section{Simulation Model}

The simulator models the kinematics and dynamics of rigid bodies in two dimensions (2-D) using the open-source Enki simulation toolkit [24]. The 2-D space 
is modeled continuously. Time progresses in discrete steps. The environment is a bounded squared world of side length $250 \mathrm{~cm}$. At any moment in time, it is partitioned into distinct regions where light is either present (day regions) or not present (night regions). The world is populated by physical objects (modules or composite entities). The objects cannot move on their own, but float passively on the ground. Kinetic energy is provided by the flow of air. The flow is composed of two components: a flow in random directions of velocity $280 \mathrm{~cm} / \mathrm{s}$ and a counter-clockwise circular flow of velocity $160 \mathrm{~cm} / \mathrm{s}$ around the world's center. The forces exerted by the flow of air result in random or circular motion patterns. In nature, such motion patterns could result from ocean currents, gravitational fields, or Brownian motion. The combination of circular motion patterns with a squared world is expected to provide nonlinearity to the dynamics of the system.

The system's basic building blocks, the modules, are modeled as squares of side length $7 \mathrm{~cm}$ and of mass $49 \mathrm{~g}$. The modules can physically connect with each other and thereby form composite entities. Each module controls the connectivity of each of its four sides by activating or deactivating it. If the sides of two separate modules are well aligned with each other, a connection is established provided that at least one of the two sides is activated.

The system has three types of modules:

1. The energy module, or $e$-module, harvests, stores, and provides energy. The energy consumption is 1 unit/s for modules of all types. When part of a same composite entity, e-modules share instantaneously their energy with all other modules via a power line. They also balance their energy storage over time.

2. The interaction module, or $i$-module, allows composite entities to respond to stimuli in their environment. In this study, the $i$-module can (i) adjust the friction (coefficient) it has by contact with the ground and (ii) perceive whether it is located in a day or night region. The module's behavior is hard-wired as follows: the friction coefficient is 0.2 in day regions, and it is 0.02 in night regions or whenever the module is powered off. The $i$-module is powered on whenever it receives energy through its power lines.

3 . The boundary module, or $b$-module, allows composite entities to be encapsulated by a protecting membrane (boundary). Once a $b$-module has attached, the composite entity is prevented from further growth. The $b$-module is powered on whenever it receives energy through its power lines.

\section{Origin of Organisms, Replication, and Variation}

At the beginning of a trial, the modules are randomly distributed in the world (see Fig. 1a). When an $e$-module retrieves energy from the environment, it gets automatically powered on. It then activates all four of its connection sides. Recall that a connection between two modules can be established only if at least one of the interacting connection sides is active. As the $i$ - and $b$ - modules do not have energy on their own, at this stage any growth is seeded by at least one $e$-module. When a separate $e$ - or $i$ - module connects with another module during the 


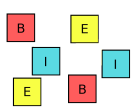

(a)

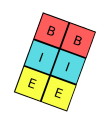

(c)

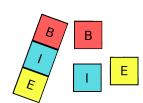

(b)

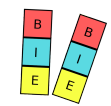

(d)
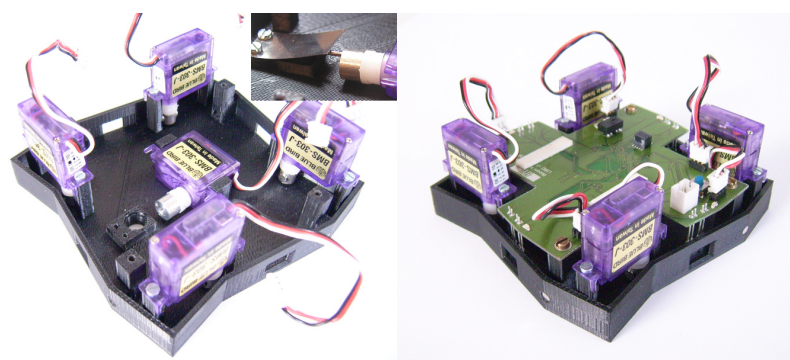

Fig. 1. Left: $(\mathrm{a}-\mathrm{d})$ : illustration of the growth and replication process. Center/right: generic hardware prototype capable of simulating all aspects of the $e-, i-$, and $b$ modules. Center: squared base with four connection sides. The inset shows a hatch in the bottom plate, which controls the mobility of the module, which floats on an air table. Right: fully assembled prototype (battery removed).

growth phase, it deactivates its two lateral connection sides. As a consequence, the modules form polymers (i.e., linear chains) of arbitrary length. Once a $b$ module connects to either end of such a polymer, a signal propagates to the other end, and thereby a membrane is established (see Fig. 1b). The membrane prevents further extensions on either side of the polymer during its lifetime. The self-assembly process thus results in linear organisms that are composed of $\geq 1$ $e$-modules, $\geq 0 i$-modules, and $1 b$-module.

Organisms attempt to replicate at any moment in time. To do so, the $b$ module activates one lateral connection side. Once a module of correct type has attached to this side, the $b$-module sends a signal to the next module in the organism chain. The replication process then proceeds by copying elements, one by one, similar to the Watson-Crick base pairing. In our case, modules pair only with modules of the same type (see Fig. 1c). Mismatches in type are recognized and the modules released. Once the replication has completed, the two organisms split apart (see Fig. 1d).

In some situations composite entities (including organisms) break apart. First, this happens when a composite entity has no energy left (i.e., selection occurs via the environment). Second, this can happen when a module of a composite entity detects a local inconsistency. For example, this is the case when two $b$-modules connect at about the same time to both ends of a polymer. Third, composite entities can break apart when experiencing a high impulse during collision. The composite entity then splits into two or more parts. The aforementioned situations can lead to variations of the organisms (and composite entities). The resulting composite entities can form new polymer structures (if lacking a membrane), for example, by recombining with each other.

The exact logic governing the local interactions during growth and selfreplication is coded in the form of finite state machines (FSM). The entire process is regulated by local information only. Communication between two adjacent modules is limited to a single byte per time step in each direction (regardless of the size of the composite entity). 


\section{Results}

To assess our system, we put 150 modules - 50 per type - at random positions in the world. At the beginning, the energy storage of each $e$-module was empty. Its capacity was limited to 300 units. When powered, modules of all types consumed 1 unit/s. Energy was provided at a rate of 1.41 units/s by a single day region covering the entire world. After $36000 \mathrm{~s}(10 \mathrm{~h})$ had elapsed, energy was provided at a rate of $1.90 \mathrm{units} / \mathrm{s}$ by three non-overlapping day regions covering each a $7 / 36$ th circular segment of the entire world. The day regions were separated by equally-sized night regions (i.e., $5 / 36$ th circular segments of the world), which provided no energy. The day and night regions moved at a constant speed similar to the sun relative to the Earth. If a module could remain motionless it would experience a "sunrise" every 1200 s followed by a "sunset" 700 s thereafter. The circular air flow, which was driving the modules, was exactly opposing the circular movement of the day and night regions.

Figure 2 (left) shows a snapshot taken from an experiment at time 2700 s. In general, almost all of the organisms that emerged were not capable of harvesting enough energy to stay alive; typically they died shortly after becoming alive or when reproducing. In the first phase $(10 \mathrm{~h})$, the energy was uniformly distributed in the world. Consequently, mobility was not relevant for energy retrieval. This certainly explains the lack of $i$-modules (which control ground friction as a response to light) in the organisms that evolved at this stage [see Fig. 2 (center)]. We repeated the phase 1 evolution ten times and in all cases a few organisms of adequate structure emerged spontaneously and then replicated rapidly until the initial supply of modules was exhausted. In the evolutionary run shown in Fig. 2 (center/right), the population converged to a single species represented by eight identical individuals (seven of which were generated by self-replication). Each individual consisted of $4 e$-modules and $1 b$-module. The remaining $18 e$-modules were attached to these organisms in the form of base pairs.

In the second phase, which started when 36000 s (10 h) had elapsed, energy was not uniformly distributed. At the beginning of phase 2, we observed the extinction of the aforementioned species [Fig. 2 (right)]. During phase 2 new organisms emerged. The most successful ones comprised $6 e$-modules, $1 i$-module, and $1 b$-module. The $i$-modules enabled the organisms to increase the relative time spent within the day regions. Preliminary analysis suggests that the position of the $i$-module within the organism was also a crucial factor for survival. As can be seen in Fig. 2 (right), some of the organisms in phase 2 were produced by self-replication. However, different from phase 1 these organisms did not spread in the entire population. A possible explanation for this is that the mean density of energy had dropped from 1.41 units/s in phase 1 to $1.108 \overline{3}$ units/s in phase 2 . Recall that 1 unit/s is consumed already by the $e$-module itself. In addition, the environment in phase 2 was highly unpredictable as energy was supplied only in certain regions, which changed over time. The limited size of these regions certainly created competition between the organisms, which - due to their embodiment - could not occupy the same positions. 

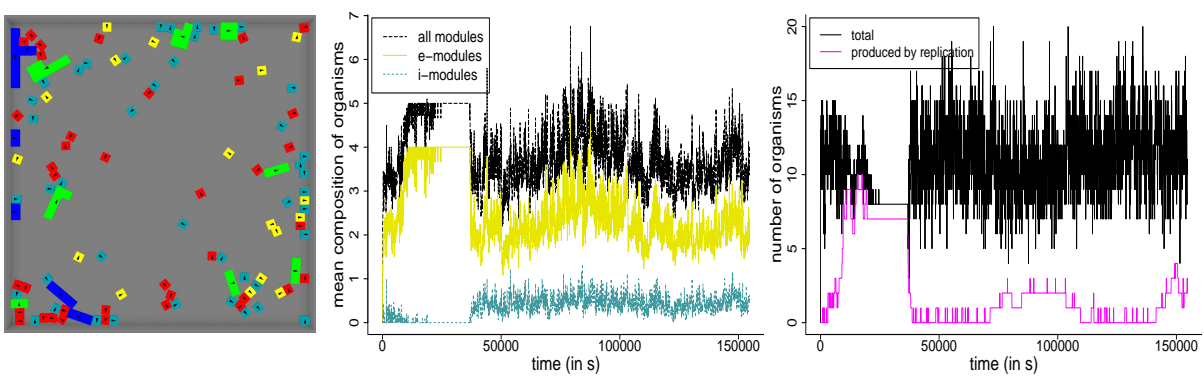

Fig. 2. Left: snapshot taken in a simulation trial (green: organisms, some of which are replicating, blue: polymers without membrane, yellow/turquoise/red: $e / i / b$-modules). Center: mean composition (i.e., modular makeup) of organisms over time. Right: total number of organisms and number of those produced by replication over time.

\section{Hardware Implementation}

We have designed and built a generic hardware prototype, which can simulate all aspects of the $e^{-}, i-$, and $b$-modules. The prototype is shown in Fig. 1 (center/right). It has a size of $7 \mathrm{~cm}$ times $7 \mathrm{~cm}$, a total weight of $59 \mathrm{~g}$, and can float on an air table. The module's base [see Fig. 1 (center)] was fabricated using a 3D printer. Its slanted edges facilitate self-alignment when colliding with other modules. A module can attach to other modules on each of its four sides. The connection mechanism is similar to the one reported by Klavins' group in [25]. Each side has two permanent magnets. One magnet is fixed in position with the north pole pointing outwards. The other magnet can be rotated by means of a servomotor, which gives basic control on the level of attraction or repulsion. A hatch in the base can be opened or closed in order to allow the module to immobilize itself: when the hatch is opened, air from the table flows through the opening and as a consequence the module's ground friction increases. The hatch is actuated by a fifth servomotor, which lies flat on the base. The module contains a printed circuit board with a dsPIC33F microcontroller. For inter-module communication, the module has four infrared transmitters. A fifth transmitter (light sensor) is mounted on top of the module, pointing upwards. A $350 \mathrm{mAh}$ lithium polymer battery provides energy.

\section{Discussion}

In this paper, we proposed a self-assembling system that can allow non-biological evolutionary processes to take place in the physical world. The evolutionary process is fully autonomous, decentralized, and self-organized. It is governed by the organisms' physical interactions with each other and with their environment. The physical interactions are in turn determined by a number of factors. For example, the motion of an organism (which is made possible by the flow of air in the environment) is affected by the organism's mass, center of mass, moment of 
inertia, the orientation-dependent surface area, geometry, and the non-uniform friction - all parameters that vary with the organism's modular makeup.

First results obtained in computer simulations are very promising and indicate the feasibility of such evolutionary process. In particular, the system proved capable of generating a population of organisms that had qualities of living beings (e.g., response to stimuli) and that were well adapted to their environment, even when the latter was subject to gradual or sudden changes. Clearly, most of the system's behavior is yet to be explored. For example, we suppose that the role of variations were very limited in the experiments we reported (only a few organisms broke apart and recombined to new solutions). However, we expect the role of variation to become more important when the environment complexity and the organisms' sizes further increase - under these circumstances it should be more difficult to assemble an appropriate solution from scratch.

We have constructed a hardware prototype that implements the required key functionalities apart from energy sharing and energy retrieval. In principle, these functionalities can be simulated in a physical setting by using the prototype's

onboard battery and light sensor. As onboard batteries will limit the time of operation, we plan to equip the modules with solar panels and energy sharing facilities [26]. The system should then be capable of exhibiting an autonomous physical evolution in a human designed environment. The ultimate goal would be to design a system that can evolve physical organisms in natural environments.

\section{Acknowledgment}

The authors thank Prof. Jarle Breivik for stimulating discussions that helped in the preparation of the manuscript. We thank Tarek Baaboura, Daniel Burnier, and Philippe Rétornaz for help in building the hardware prototype. This work was supported by the Sixth Framework Programme of the European Community in the form of a Marie Curie Intra-European Fellowship (contract no. MEIF-CT2006-040312). It reflects only the authors' views. The European Community is not liable for any use that may be made of the information.

\section{References}

1. Pfeifer, R., Bongard, J.C.: How the body shapes the way we think. MIT Press, Cambridge, MA (2006)

2. Floreano, D., Mondada, F.: Automatic creation of an autonomous agent: Genetic evolution of a neural-network driven robot. In: Proc. of the 3rd Int. Conf. on Simulation of Adaptive Behavior (SAB'94), MIT Press, Cambridge, MA (1994) $421-430$

3. Thompson, A.: Artificial evolution in the physical world. In: Proc. of the 5th Int. Symp. on Evolutionary Robotics (ER'97), AAI Books (1997) 101-125

4. Sims, K.: Evolving virtual creatures. In: Proc. of the 21st Annu. Conf. on Comput. Graphics and Interactive Tech. (SIGGRAPH), ACM Press, New York (1994) 15-22

5. Sims, K.: Evolving 3D morphology and behavior by competition. Artif. Life 1(4) (1994) 353-372 
6. Taylor, T., Massey, C.: Recent developments in the evolution of morphologies and controllers for physically simulated creatures. Artif. Life 7(1) (2000) 77-87

7. Funes, P., Pollack, J.: Evolutionary body building: Adaptive physical designs for robots. Artif. Life 4(4) (1998) 337-357

8. Lipson, H., Pollack, J.B.: Automatic design and manufacture of robotic lifeforms. Nature 406(6799) (2000) 974-978

9. Chou, H., Reggia, J.A.: Emergence of self-replicating structures in a cellular automata space. Physica D 110(3-4) (1997) 252-276

10. Dittrich, P., Banzhaf, W.: Self-evolution in a constructive binary string system. Artif. Life 4(2) (1998) 203-220

11. Watson, R.A., Ficici, S.G., Pollack, J.B.: Embodied evolution: Distributing an evolutionary algorithm in a population of robots. Robot. Auton. Syst. 39(1) (2002) $1-18$

12. Bianco, R., Nolfi, S.: Toward open-ended evolutionary robotics: Evolving elementary robotic units able to self-assemble and self-reproduce. Connection Science 16(4) (2004) 227-248

13. Channon, A.: Unbounded evolutionary dynamics in a system of agents that actively process and transform their environment. Genet. Program. Evolvable Mach. 7(3) (2006) 253-281

14. Studer, G., Lipson, H.: Spontaneous emergence of self-replicating structures in molecube automata. In: Proc. of the 10th Int. Conf. on the Simulation and Synthesis of Living Systems (Artificial Life X), MIT Press, Cambridge, MA (2006) $227-233$

15. Smith, A., Turney, P., Ewaschuk, R.: Self-replicating machines in continuous space with virtual physics. Artif. Life 9(1) (2003) 21-40

16. Whitesides, G.M., Boncheva, M.: Beyond molecules: Self-assembly of mesoscopic and macroscopic components. Proc. Natl. Acad. Sci. U.S.A. 99(8) (2002) 47694774

17. Groß, R., Dorigo, M.: Self-assembly at the macroscopic scale. Proceedings of the IEEE 96(9) (2008) 1490-1508

18. Penrose, L.S., Penrose, R.: A self-reproducing analogue. Nature 179(4571) (1957) 1183

19. Jacobson, H.: On models of reproduction. Am. Sci. 46 (September 1958) 255-284

20. Zykov, V., Mytilinaios, E., Adams, B., Lipson, H.: Self-reproducing machines. Nature 435(7039) (2005) 163-164

21. Griffith, S., Goldwater, D., Jacobson, J.M.: Self-replication from random parts. Nature 437(7059) (2005) 636

22. Christensen, A.L., O'Grady, R., Dorigo, M.: SWARMORPH-script: A language for arbitrary morphology generation in self-assembling robots. Swarm Intell. 2(2-4) (2008) 143-165

23. Breivik, J.: Self-organization of template-replicating polymers and the spontaneous rise of genetic information. Entropy 3(4) (2001) 273-279

24. Magnenat, S., Waibel, M., Beyeler, A.: Enki: The fast 2D robot simulator. http: //home.gna.org/enki/ (2009)

25. Bishop, J., Burden, S., Klavins, E., Kreisberg, R., Malone, W., Napp, N., Nguyen, T.: Programmable parts: A demonstration of the grammatical approach to selforganization. In: Proc. of the 2005 IEEE/RSJ Int. Conf. on Intelligent Robots and Systems, IEEE Computer Society Press, Los Alamitos, CA (2005) 3684-3691

26. White, P., Zykov, V., Bongard, J., Lipson, H.: Three dimensional stochastic reconfiguration of modular robots. In: Proc. of the 2005 Robotics: Science and Systems Conf., MIT Press, Cambridge, MA (2005) 161-168 